\title{
Species of Chrysotus Meigen in Palaearctic China (Diptera: Dolichopodidae)
}

\author{
Mengqing Wang \& Ding Yang*
}

Wang, M. \& Yang, D. 2008: Species of Chrysotus Meigen in Palaearctic China (Diptera: Dolichopodidae). — Entomol. Fennica 19: 232-240.

The species of the genus Chrysotus from Palaearctic China are reviewed and keyed. The following 5 species are described as new to science: Chrysotus bifurcatus sp. n., C. brevicercus sp. n., C. luoyangensis sp. n., C. sinuolatus sp. n., and C. xinjiangensis sp. $\mathrm{n}$. Three species are recorded from China for the first time: $C$. gramineus (Fallén), C. suavis Loew, and C. pennatus Lichtwardt. Information on their distribution is also given or updated.

M. Q. Wang, Institute of Plant Protection, Chinese Academy of Agricultural Sciences, Beijing 100094, China

D. Yang (*author for correspondence), Department of Entomology, China Agricultural University, Beijing 100094, China; E-mail: dyangcau@yahoo.com.cn

Received 11 June 2007, accepted 16 November 2007

\section{Introduction}

The genus Chrysotus Meigen, 1824 belongs to the subfamily Diaphorinae. It can be easily recognized from other Diaphorinae species by the following characters: male and female frons wider than face; eyes shortly haired; male eyes with enlarged facets toward face; first flagellomere of male with arista subapical in notch or to side of tip; upper part of propleuron bare; male abdominal tergite 6 with bristles; surstylus not branched, tapering and curved apically (Robinson \& Vockeroth 1981, Wang \& Yang 2006). It is distributed worldwide with 285 described species (Yang et al. 2006), of which 55 species are known from the Palaearctic, 17 from the Oriental, 20 from the Australian, 13 from the Afrotropical, 107 from the Nearctic, and 92 from the Neotropical region. Negrobov and his co-workers (1980, 1995, 2000, and 2003) revised the Palaearctic Chrysotus.

In the present paper, five new species are described, three species are recorded from China for the first time, and a key to the species of the genus from Palaearctic China is presented to separate these species.

\section{Materials and methods}

The specimens on which this study are based were collected from Henan, Hebei, Shandong, Beijing, Gansu, Qinghai, Ningxia, Neimeng, Xinjiang and Jilin by different collectors in different years. All material is preserved in $75 \%$ alcohol and deposited in the Entomological Museum of China Agricultural University, Beijing.

Morphological terminology for adult structures mainly follows McAlpine (1981). Terms for the structures of the male genitalia follow Cumming et al. (1995). The abbreviations are used as follows: acr $=$ acrostichal bristle, $\mathrm{ad}=$ anterodorsal bristle, av $=$ anteroventral bristle, $\mathrm{CuAx}$ ratio $=$ length of $\mathrm{m}-\mathrm{cu} /$ length of distal portion of $\mathrm{CuA}, \mathrm{d}=$ dorsal bristle, $\mathrm{dc}=$ dorsocentral 
bristle, $\mathrm{LI}=$ fore leg, $\mathrm{LII}=$ mid leg, LIII = hind leg, $\mathrm{pd}=$ posterodorsal bristle, $\mathrm{pv}=$ posteroventral bristle, $\mathrm{v}=$ ventral bristle.

\section{Taxonomy}

\subsection{Key to species of Chrysotus from Palaearctic China (males)}

1. Fore coxa with all hairs and bristles pale 2

- Fore coxa at least with bristles black

2. Hind tarsus pale yellow 10

- Hind tarsus black or dark brown denticles apically. Beijing

serratus Wang \& Yang, 2006

- Mid femur pale yellow; aedeagus without rowed denticles apically

4. Hind femur wholly yellow

- Hind femur with black apex. Jilin, Ningxia, Neimeng, Hebei, Shanxi, Tianjin, Shandong cilipes Meigen, 1824

5. First flagellomere large with blunt apex, nearly square. Beijing, Hebei liui nom. n.

- First flagellomere small with acute apex, subtriangular. Beijing, Henan, Taiwan degener Frey, 1917

6. Fore and mid tibiae yellow

- All tibiae black. Hebei

laesus (Wiedemann, 1817)

7. Mid tibia with 3 ad and 2 pd bristles. Henan uoyangensis sp. $\mathrm{n}$.

- Mid tibia with 1 or 2 ad bristles, but without pd bristle

8

8. Hind tibia and tarsus without flattened bristle; aedeagus with large symmetrically bifurcated apical portion. Xinjiang, Beijing

suavis Loew, 1857

- Hind tibia and tarsus with 2 rows of dense flattened long bristles; aedeagus not as above

9. Squama with black hairs; aedeagus with furcated apex. Hebei, Beijing

pennatus Lichtwardt, 1902

- Squama with pale yellow hairs; aedeagus not furcated apically. Liaoning rubzovi Negrobov \& Maslova, 1995
10. Hind tarsus chiefly pale yellow

- Hind tarsus chiefly or entirely brown to black 14

11. Mid femur chiefly pale yellow 12

- Mid femur chiefly brown or black 13

12. Hind tibia black. Jilin, Hebei femoratus Zetterstedt, 1843

- Hind tibia chiefly pale yellow. Jilin, Xinjiang, Ningxia, Gansu, Neimeng, Beijing, Shandong, Fujian

pseudocilipes Hollis, 1964

13. Mid tibia with $1 \mathrm{~d}$ bristle; aedeagus with furcated apical portion. Gansu, Guizhou

bifurcatus sp. n.

- Mid tibia with $2 \mathrm{~d}$ bristles; apical portion of aedeagus not furcated. Gansu, Shaanxi, Shanxi, Hebei, Beijing, Guizhou pulchellus Kowarz, 1874

14. Fore coxa yellow, mid and hind coxae black

- All coxae black or blackish

15. Hind femur yellow with black apex, hind tibia entirely black; aedeagus apically with 2 swollen lateral lobes. Beijing xiaolongmensis Wang \& Yang, 2006

- Hind femur and tibia yellow; aedeagus apically with 1 swollen lateral lobe. Xinjiang xinjiangensis $\mathrm{sp} . \mathrm{n}$.

16. First flagellomere with blunt apex, nearly trapeziform; aedeagus apically with curved thin process. Qinghai sinuolatus sp.n.

- First flagellomere with acute apex, subtriangular; aedeagus apically without curved thin process

17. Mid and lower postocular bristles pale yellow. Gansu, Shaanxi, Shanxi, Hebei, Guizhou gramineus (Fallén, 1823)

- Mid and lower postocular bristles black 18

18. First flagellomere 1.3 times wider than long; hind femur without rowed $\mathrm{v}$ bristle; hind tibia with row of $v$ bristles. Henan, Shandong brevicercus sp. $\mathrm{n}$.

- First flagellomere 2 times wider than long; hind femur with row of long $\mathrm{v}$ bristles; hind tibia without rowed $\mathrm{v}$ bristle. Beijing beijingensis Wang \& Yang, 2006 


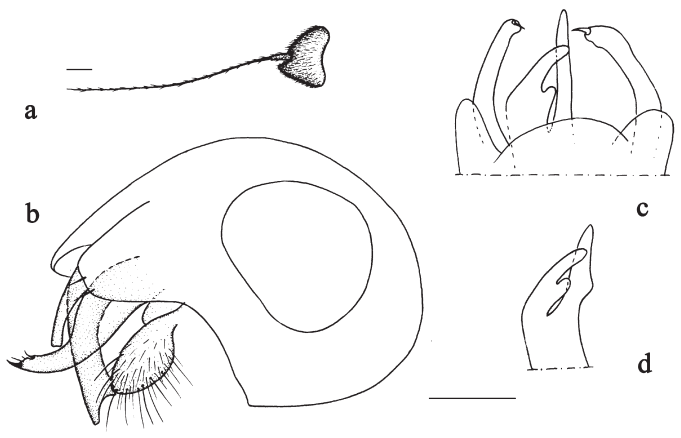

Fig. 1. Chrysotus bifurcatus sp. n., male. - a. First flagellomere, lateral view. - b. Genitalia, lateral view. c. Tip of genitalia, ventral view. $-d$. Aedeagus, ventral view. Scale bar $0.1 \mathrm{~mm}$.

\subsection{Chrysotus bifurcatus sp. n. (Fig. 1)}

Material examined. Holotype $\widehat{\jmath}$ : Gansu: Wenxian, 7.VIII.1980, leg. Ch. K. Yang. Paratypes $1 \hat{\jmath}, 1$ : : same data as holotype; $1 \hat{\jmath}, 1$ : Guizhou: Huaxi 1,000 m a.s.1., 27.V.1980, leg. Ch. K. Yang.

Diagnosis. Eyes contiguous on face. First flagellomere subtriangular, as long as wide. Legs brown except all coxae blackish, fore and mid femora with yellow apical portion, and all tibiae yellow. Aedeagus with bifurcated apex.

Description. Male. Body length 2.1-2.5 mm, wing length 1.9-2.0 mm.

Head metallic green with pale gray pollen; eyes contiguous on face. Hairs and bristles on head black, postocular bristles (including postero-ventral hairs) pale. Antenna black; first flagellomere subtriangular (Fig. 1a), as long as wide; arista black, short pubescent. Proboscis brownish with brown hairs; palpus yellow with 1 black apical bristle.

Thorax dark metallic green with pale gray pollen. Hairs and bristles on thorax black; 5 strong dc, 6-7 irregularly paired acr short and hair-like; scutellum with 2 pairs of bristles, basal pair short and hair-like; propleuron with 1 black hair and 1 black bristle on lower part.

Legs brown, all coxae blackish; fore and mid femora with yellow apical portion, all tibiae yellow, all tarsi from tip of tarsomere 1 onward pale yellow to dark yellow. Hairs and bristles on legs brown. Fore and mid coxae with brown antero- apical bristles; hind coxa with 1 outer bristle at base. Fore femur with 3-4 pv apically; mid femur with 2-3 av apically; hind femur with $2-3$ av and 2 pv apically. Fore tibia with 1 ad at basal 1/4, apically with comb-like hairs and 3 bristles; mid tibia with 1 ad, apically with 4 bristles; hind tibia with $1 \mathrm{ad}$ and $1 \mathrm{pd}$, apically with comb-like hairs and 4 bristles. All tarsomere 1 with row of short v. Relative length of tibia and 5 tarsomeres of legs LI 3.4 $: 1.7: 0.8: 0.6: 0.4: 0.4 ;$ LII $4.2: 2.1: 1.0: 0.7: 0.4$ $: 0.4 ;$ LIII $4.6: 1.4: 1.0: 0.7: 0.5: 0.4$.

Wing hyaline, veins black; $\mathrm{R}_{4+5}$ and $\mathrm{M}$ parallel apically, CuAx ratio 0.3. Squama pale with yellow hairs. Halter pale.

Abdomen dark metallic green with pale gray pollen; hairs and bristles on abdomen black. Male genitalia (Figs. 1b-d): Epandrium somewhat rounded, surstylus tapering apically; cercus short and wide, with moderately long hairs; aedeagus with bifurcated apex.

Female. Body length 2.1-2.5 mm, wing length 1.9-2.0 mm. Similar to male.

Remarks. The new species is similar to Chrysotus xiaolongmensis Wang \& Yang, 2006, but may be separated from the latter by having the fore coxa blackish and hind tibia yellow, and the aedeagus is bifurcated apically. In xiaolongmensis, the fore coxa is yellow, the hind tibia is black, and the aedeagus is not furcated apically.

Distribution. China (Gansu, Guizhou).

Etymology. The specific epithet refers to the aedeagus with the bifurcated apex.

\subsection{Chrysotus gramineus (Fallén, 1823)}

Fig. 2)

Dolichopus gramineus Fallén, 1823. Monogr. Dolichopod. Svec.: 19. Type locality: not given [Sweden].

Material examined. 1§̄: Gansu: Wenxian, 7.VIII.1980, leg. Ch. K. Yang; 10̄, 1 q : Guizhou: Huaxi 1,000 m a.s.1., 27.V.1981, leg. Ch. K. Yang.

Diagnosis. Eyes contiguous on face. Middle and lower postocular bristles (including posteroventral hairs) pale yellow. First flagellomere subtriangular, 1.3 times wider than long. Acr 4-5 pairs. All coxae blackish. Hind tibia with row of av. Squama pale yellow with pale hairs, halter 


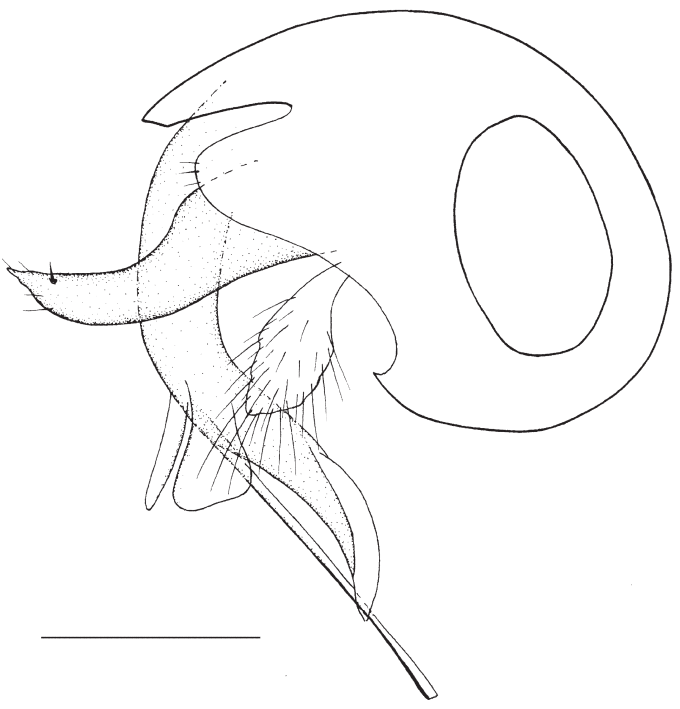

Fig. 2. Chrysotus gramineus (Fallén), male. genitalia, lateral view. Scale bar $0.1 \mathrm{~mm}$.

pale yellow. Surstylus with acute apex, and aedeagus with bifurcated apex.

Distribution. China (Gansu, Shaanxi, Shanxi, Hebei, Guizhou); England, Sweden, Finland, Ireland, Norway, Denmark, Belgium, Belarus, Germany, France, Netherlands, Austria, Switzerland, Italy, Andorra, Greece, Spain, Hungary, Bulgaria, Czech, Slovakia, Romania, Poland, Yugoslavia, Russia, Ukraine, Kirgizia, Korea.

\subsection{Chrysotus brevicercus sp. n. (Fig. 3)}

Material examined. Holotype $\hat{\delta}$ : Henan: Songxian, Baiyunshan, 18.VII.2004, leg. H. Dong. Paratypes $6 \hat{\jmath} \hat{\jmath}$ : same data as holotype;

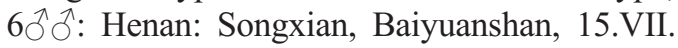
2004, leg. K. Y. Zhang; 1 $\delta^{\text {: }}$ : Henan: Nanyang, Neixiang, Baotianman, 25.VII.2004, leg. H. Dong; $2 \widehat{\jmath}$ đ: Henan: Nanyang, Neixiang, Baotianman, 24.VII.2004, leg. K. Y. Zhang; 20ðð Henan: Songxian, Tianchishan, 12-13.VII.2004, leg. K. Y. Zhang; 1 $\delta^{\Uparrow}$ : Henan: Luoyang, Luanchuan, Longyuwan, 19.VII.2004, leg. K. Y. Zhang; 10우 Henan: Luoyang, Luanchuan, Longyuwan, 21.VII.2004, leg. K. Y. Zhang; 5ภิ ภ: Henan: Luoyang, Luanchuan, Longyuwan, 20.VII.2004, leg. H. Dong; 1ठ: Shandong: Mouping, Kunyushan, 19.VIII.2004, leg. H. Dong.
Diagnosis. Eyes narrowly separated on face. First flagellomere subtriangular, 1.3 times wider than long. Legs chiefly black; fore and mid tibiae chiefly yellow with blackish bases; fore and mid tarsi from tip of tarsomere 1 onward brown to dark brown; hind tibia brownish, with basal $1 / 3$ and apical $1 / 3$ brown, hind tarsus entirely brown. Male cercus short and broad, with weak marginal processes.

Description. Male. Body length 2.2-2.5 mm, wing length $1.8-1.9 \mathrm{~mm}$.

Head metallic green with pale gray pollen; eyes narrowly separated on face. Hairs and on head black, postocular bristles (including postero-ventral hairs) black. Antenna black; first flagellomere subtriangular (Fig. 3a), 1.3 times wider than long; arista black, short pubescent. Proboscis blackish with black hairs; palpus brown with 1-2 black apical bristles.

Thorax metallic green with pale gray pollen. Hairs and bristles on thorax black; 5 strong dc, 56 irregularly paired acr short and hair-like; scutellum with 2 pairs of bristles, basal pair short and hair-like; propleuron with 2 black bristles on lower part.

Legs black; all coxae and trochanters black; fore and mid femora chiefly black with dark yellow tip; fore and mid tibiae yellow with blackish bases; fore and mid tarsi from tip of tarsomere 1 onward brown to dark brown; hind femur black, hind tibia brownish with basal $1 / 3$ and apical $1 / 3$ brown, hind tarsus entirely brown. Hairs and bristles on legs black. Fore and mid coxae with black antero-apical bristles; hind coxa with 1 black

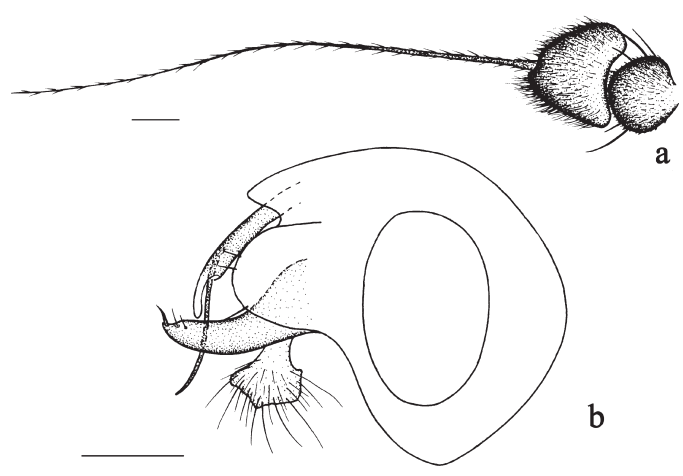

Fig. 3. Chrysotus brevicercus sp. n., male. - a. Antenna (excluding scape), lateral view. - b. Genitalia, lateral view. Scale bar $0.1 \mathrm{~mm}$. 
outer bristle at base. Fore femur with 2-3 pv apically; mid femur with 2 av and $2-3$ pv apically; hind femur with row of 4-5 long av and 2-3 short pv apically. Fore tibia with 1 weak ad at basal 1/4, apically with comb-like hairs and 4 bristles; mid tibia with 2 ad and $1 \mathrm{pd}$, apically with 4 bristles; hind tibia with 3 ad and row of $v$ (of which basal 5-6 longer), apically with comb-like hairs and 4 bristles; mid and hind tarsomere 1 each with row of short v. Relative length of tibia and 5 tarsomeres of legs LI $3.6: 1.7: 1.0: 0.8: 0.5: 0.5$; LII $4.1: 2.2: 1.0: 0.8: 0.6: 0.5 ;$ LIII $5.0: 1.5: 1.2$ : $0.8: 0.6: 0.5$.

Wing hyaline, veins brown; $\mathrm{R}_{4+5}$ and $\mathrm{M}$ parallel apically, $\mathrm{CuAx}$ ratio 0.3. Squama yellow with black hairs. Halter pale yellow.

Abdomen metallic green with pale gray pollen; hairs and bristles black. Male genitalia (Fig. 3b): Epandrium somewhat rounded, surstylus tapering and curved apically; cercus short and broad, with weak marginal processes; aedeagus slender; hypandrium short.

Female. Body length 2.8-3.1 mm, wing length $2.2-2.5 \mathrm{~mm}$. Similar to male, but eyes widely separated on face, and hind tibia without rowed $\mathrm{v}$.

Remarks. The new species is similar to Chrysotus logvinovskii Negrobov, Tsurikov et Maslova, but may be separated from the latter by the postocular bristles (including postero-ventral hairs) black and the different male genitalia. In logvinovskii, the postocular bristles (including postero-ventral hairs) are pale yellow.

Distribution. China (Henan, Shandong).

Etymology. The specific epithet refers to the short and broad cercus.

\subsection{Chrysotus liui nom. n.}

Chrysotus liui nom. n. for Chrysotus quadratus Wang \& Yang, 2006 (preoccupied by Van Duzee, 1915).

Chrysotus quadratus Wang \&Yang, 2006. Mitt. Mus. Nat. kd. Berl., Dtsch. entomol. Z. 52(2): 251. Type locality: Beijing.

Material examined. Holotype 0 : Beijing: Xiangshan Park, 17-19.VIII.1997, leg. D. Yang. Paratypes $4 \hat{\jmath}$ : same data as holotype. Other

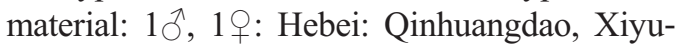

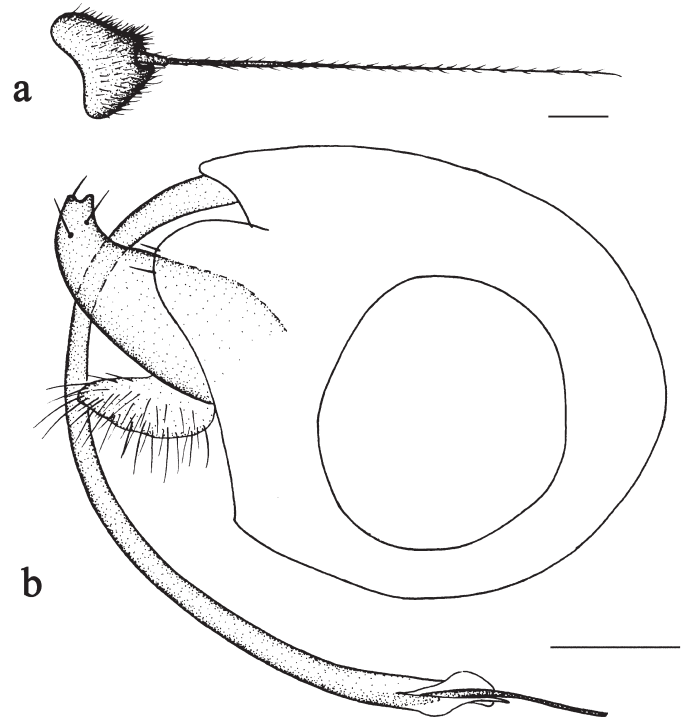

Fig. 4. Chrysotus luoyangensis sp. n., male. - a. First flagellomere, lateral view. - b. Genitalia, lateral view. Scale bar $0.1 \mathrm{~mm}$.

chang, 16.VIII.2004, leg. X. Y. Liu.

Diagnosis. Eyes very narrowly separated on face. First flagellomere somewhat quadrate. Middle and lower postocular bristles pale. Legs chiefly yellow. Fore coxa yellow with pale hairs and bristles.

Distribution. China (Beijing, Hebei).

\subsection{Chrysotus luoyangensis sp. n. (Fig. 4)}

Material examined. Holotype $\widehat{\jmath}$ : Henan: Luoyang, Luanchuan, Longyuwan 1,000m a.s.1., 19.VII.2004, leg. K. Y. Zhang. Paratypes $10^{\Uparrow}, 1$ \% Henan: Xinyang, Jigongshan, 13.VII.1997, leg. Zh. Li; $2 \hat{\jmath} \widehat{\jmath}, 1$ iq: Gansu: Chengxian, 20.VII. 1980, leg. Ch. K. Yang.

Diagnosis. Eyes contiguous on face. Legs yellow; mid and hind coxae black; hind femur with apical half blackish; hind tibia with apical 1/3 blackish; hind tarsus blackish. Aedeagus slender, furcated apically.

Description. Male. Body length 2.0-2.3 mm, wing length $1.8-2.1 \mathrm{~mm}$.

Head metallic green with pale gray pollen; eyes contiguous on face. Hairs and bristles on head black, postocular bristles (including postero-ventral hairs) pale yellow. Antenna 
black; first flagellomere 1.2 times wider than long (Fig. 4a), trapeziform; arista black, short pubescent. Proboscis blackish with black hairs; palpus yellow with 2 brown apical bristles.

Thorax metallic green with pale gray pollen. Hairs and bristles on thorax black; 5 strong dc, 56 irregularly paired acr short and hair-like; scutellum with 2 pairs of bristles, basal pair short and hair-like; propleuron with 2 black bristles on lower part.

Legs yellow; fore coxa yellow with blackish base, mid and hind coxae black with yellow apex; hind femur with apical half blackish; hind tibia with apical 1/3 blackish; fore and mid tarsi from tip of tarsomere 1 onward brown to dark brown, hind tarsus blackish. Hairs and bristles on legs black. Fore and mid coxae with pale yellow antero-apical bristles; hind coxa with 1 black outer bristle at base. Fore femur with 2-3 pv apically; mid femur with 2 av and 2-3 pv apically; hind femur with 3-4 av and 1-2 pv apically. Mid tibia with 3 ad (1 short and other 2 long) and 2 short pd, apically with 4 bristles; hind tibia with 1 ad, 4-5 pd, and row of av, apically with comb-like hairs and 4 bristles. Mid and hind tarsomere 1 with row of short $v$. Relative length of tibia and 5 tarsomeres of legs LI $3.6: 1.8: 0.9: 0.7: 0.4: 0.4$; LII $4.6: 2.6: 1.2: 0.9: 0.5: 0.4$; LIII $5.4: 1.5: 1.4$ : $0.9: 0.5: 0.5$.

Wing hyaline, veins black; $\mathrm{R}_{4+5}$ and $\mathrm{M}$ parallel apically, $\mathrm{CuAx}$ ratio 0.2. Squama pale with black hairs. Halter pale.

Abdomen metallic green with pale gray pollen; hairs and bristles on abdomen black. Male genitalia (Fig. 4b): Epandrium somewhat rounded, surstylus tapering and curved apically; cercus short and wide, with somewhat acute apex; aedeagus slender, furcated apically.

Female. Body length $2.2 \mathrm{~mm}$, wing length 2.1 $\mathrm{mm}$. Similar to male.

Remarks. The new species is similar to Chrysotus decipiens Negrobov, Tsurikov et Maslova, but may be separated from the latter by the lower postocular bristles (including postero-ventral hairs) pale yellow. In decipiens, the postocular bristles (including postero-ventral hairs) are black.

Distribution. China (Henan).

Etymology. The specific epithet refers to the locality of the holotype Luoyang.

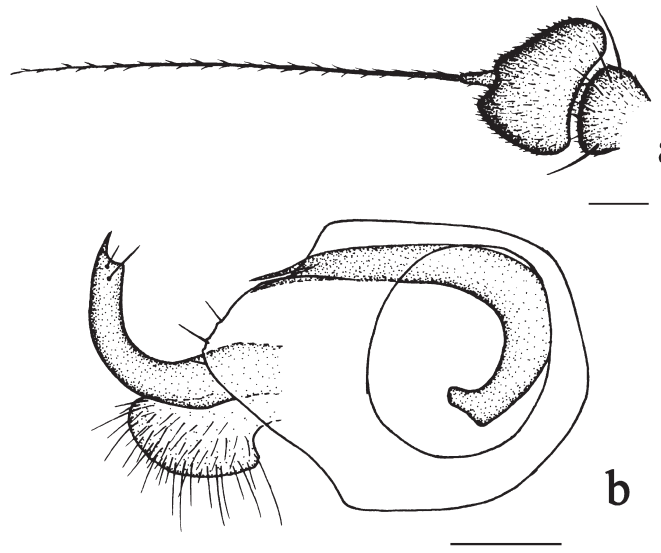

Fig. 5. Chrysotus pennatus Lichtwardt, male. - a. antenna (excluding scape), lateral view. - b. genitalia, lateral view. Scale bar $0.1 \mathrm{~mm}$.

\subsection{Chrysotus pennatus Lichtwardt, 1902} (Fig. 5)

Chrysotus pennatus Lichtwardt, 1902. Természetr. Füz. 25: 197. Type locality: Yugoslavia (Novi).

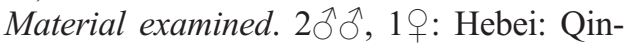
huangdao, Xiyuchang, 16.VIII.2004, leg. X. Y.

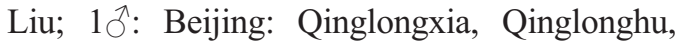
6.VII.2004, leg. Y. L. Xu; $1{ }^{\Uparrow}$ : Beijing: Gongzhufen, 2.VIII.1957, leg. Ch. K. Yang.

Diagnosis. Eyes contiguous on face. Postocular bristles (including postero-ventral hairs) pale yellow. Legs black; all coxae black; fore and mid femora with base and apex yellow, hind femur with basal portion yellow; fore and mid tibia yellow, hind tibia and tarsus black; hind tibia somewhat swollen, with row of strong and row of flattened strong ad. Aedeagus bearing symmetrically bifurcated apex.

Distribution. China (Hebei, Beijing); Germany, Italy, Greece, Hungary, Yugoslavia, Romania, Russia.

\subsection{Chrysotus sinuolatus sp. n. (Fig. 6)}

Material examined. Holotype $\widehat{\jmath}$ : Qinghai: Menyuan, Fengzhakou, 15-18.VII.1989, leg. M. C. Wei. Paratypes $24 \widehat{\jmath}, 6$, 9 : same data as holotype. 

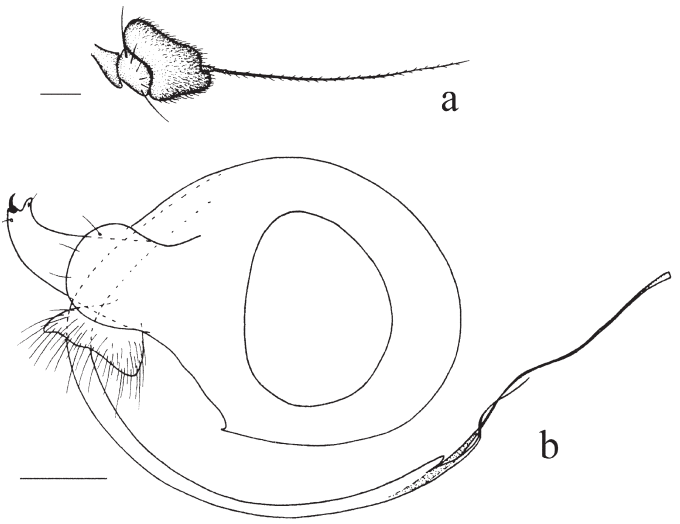

Fig. 6. Chrysotus sinuolatus sp. n., male. $-a$. Antenna, lateral view. - b. Genitalia, lateral view. Scale bar $0.1 \mathrm{~mm}$.

Diagnosis. Eyes narrowly separated on face. Legs chiefly black; fore and mid femora with yellow apex; fore and mid tibiae yellow; fore and mid tarsi from tip of tarsomere 1 onward brownish to brown, hind tarsus entirely black. Aedeagus apically with curved thin process.

Description. Male. Body length 1.8-2.2 mm, wing length 2.2-2.5 mm.

Head metallic green with pale gray pollen; eyes narrowly separated on face. Hairs and bristles on head black, lower postocular bristles (including postero-ventral hairs) pale. Antenna black; first flagellomere (Fig. 6a) large, trapeziform, as long as wide; arista black, short pubescent. Proboscis dark brown with black hairs; palpus yellow with 2 brown apical bristles.

Thorax metallic green with pale gray pollen. Hairs and bristles on thorax black; 6 strong dc, 910 irregularly paired acr short and hair-like; scutellum with 2 pairs of bristles, basal pair short and hair-like; propleuron with 1 black bristle and 1 black hair on lower part.

Legs black; all coxae black; fore and mid femora with yellow apex, hind femur black; fore and mid tibiae yellow, hind tibia black; fore and mid tarsi from tip of tarsomere 1 onward brownish to brown, hind tarsus entirely black. Hairs and bristles on legs black. Fore and mid coxae with black antero-apical bristles; hind coxa with 1 black outer bristle at base. Fore femur with $2-3$ pv apically; mid femur with 2 preapical bristles and 2-3 av apically; hind femur with 1 preapical bristle and $2-3$ av apically. Fore tibia with 1 ad at basal $1 / 4$, apically with comb-like hairs and 3 bristles; mid tibia with 2 long ad and 2-3 pd, apically with 3 bristles; hind tibia with 2 ad, 3-4 pd, and $4-5 \mathrm{v}$, apically with comb-like hairs and 3 bristles. Fore and hind tarsomere 1 with row of short v. Relative length of tibia and 5 tarsomeres of legs LI $3.3: 1.7: 0.7: 0.6: 0.4: 0.4 ;$ LII $4.2: 2.2$ : $1.0: 0.7: 0.4: 0.4 ;$ LIII $5.0: 1.5: 1.1: 0.7: 0.5$ : 0.5 .

Wing hyaline, veins black; $\mathrm{R}_{4+5}$ and $\mathrm{M}$ parallel apically, CuAx ratio 0.2. Squama yellow with black hairs. Halter pale.

Abdomen metallic green with pale gray pollen; hairs and bristles on abdomen black. Male genitalia (Fig. 6b): Epandrium somewhat rounded, surstylus wide with swollen apex; cercus with acute apex, with moderately long hairs; aedeagus slender, apically with curved thin process.

Female. Body length 1.6-1.7 mm, wing length 1.8-2.0 mm. Similar to male, but eyes widely separated on face.

Remarks. The new species is similar to Chrysotus beijingensis Wang \& Yang, 2006, but may be separated from the latter by the large first flagellomere as long as wide and surstylus with the swollen apex. In beijingensis, the first flagellomere is rounded, 2.0 times wider than long, and the surstylus has no swollen apex.

Distribution. China (Qinghai).

Etymology. The specific epithet refers to the aedeagus apically with the curved thin process.

a

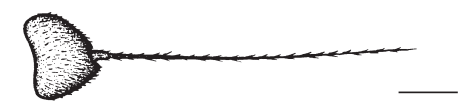

b

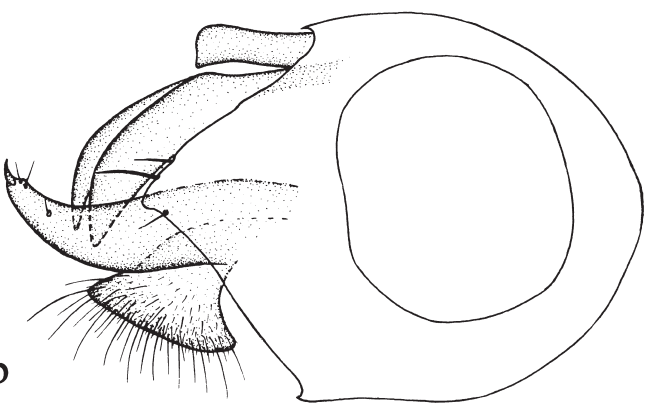

Fig. 7. Chrysotus suavis Loew, male. - a. First flagellomere, lateral view. - b. Genitalia, lateral view. Scale bar $0.1 \mathrm{~mm}$. 


\subsection{Chrysotus suavis Loew, 1857 (Fig. 7)}

Chrysotus suavis Loew, 1857. Programm K. Realschule Meseritz 1857: 49. Type locality: Germany: "Cöln"; Austria: "Neusiedler See in Ungarn”.

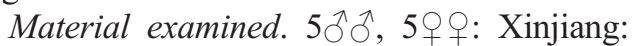
Kuerle 1,020 m a.s.1., 16.VIII.1958, leg. Ch. Q. Li; 1ð, 2우: Xinjiang: Kuerle 1,000 m a.s.l., 18.VIII.1958, leg. Ch. Q. Li; 1 ${ }^{\Uparrow}$ : Xinjiang: Tulufan, 28.VII.1958, leg. Ch. Q. Li; $1 \delta^{\Uparrow}$ : Beijing: Huairou, Qinglonghu, 6.VII.2004, leg. Y. L. Xu.

Diagnosis. Eyes contiguous on face. First flagellomere subtriangular. Legs dark brown; all femora with yellow apex, fore tibia yellow; hind tibia somewhat swollen with $4 \mathrm{pd}$. Fore and mid coxae with yellow hairs and bristles. Epandrium of male genitalia with acute apex, surstylus wide with acute apex; cercus subtriangular, with moderately long hairs; aedeagus broad, apical portion symmetrically bifurcated.

Distribution. China (Xinjiang, Beijing); Finland, England, Sweden, Norway, Switzerland, France, Belgium, Netherlands, Germany, Austria, Italy, Spain, Greece, Poland, Hungary, Romania, Bulgaria, Czech, Slovakia, Yugoslavia, Russia, Armenia, Ukraine, Middle Asia, Afghanistan, Mongolia, Iraq, Egypt, Israel, Turkey, Algeria.

\subsection{Chrysotus xinjiangensis sp. n. (Fig. 8)}

Material examined. Holotype $\hat{0}$ : Xinjiang: Urumchi, 12.VII.2005, leg. X. Sh. Bai. Paratypes

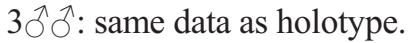

Diagnosis. Eyes contiguous on face lower portion. Legs chiefly yellow. Aedeagus slender, apically with 1 swollen lateral lobe.

Description. Male. Body length 3.0-3.6 mm, wing length $2.8-3.0 \mathrm{~mm}$.

Head metallic green with pale gray pollen; eyes contiguous on face lower portion. Hairs and bristles on head black, lower postocular bristles (including postero-ventral hairs) pale. Antenna black; first flagellomere 1.2 times wider than long; arista black, short pubescent. Proboscis brownish with brown hairs; palpus pale yellow with black hairs.

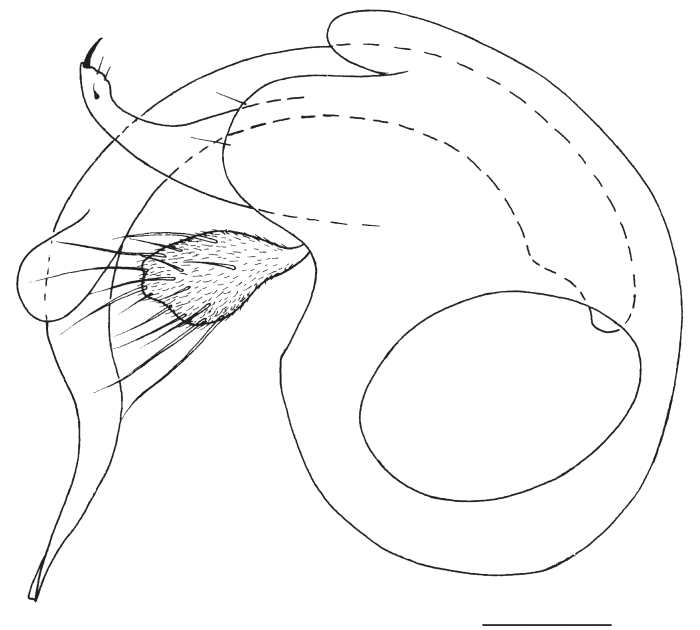

Fig. 8. Chrysotus xinjiangensis sp. n., male. genitalia, lateral view. Scale bar $0.1 \mathrm{~mm}$.

Thorax metallic green with pale gray pollen. Hairs and bristles on thorax black; 6 strong dc, 9 10 irregularly paired acr short and hair-like; scutellum with 2 pairs of bristles, basal pair short and hair-like; propleuron with 1 black bristle on lower part.

Legs yellow; fore coxa yellow with dark base, mid and hind coxae blackish; fore and mid tarsi from tarsomere 3 onward brownish to brown, hind tarsus from tip of tarsomere 1 onward brown to dark brown. Hairs and bristles on legs black. Fore and mid coxae with black antero-apical bristles; hind coxa with 1 black outer bristle at base. Fore femur with 3-4 pv apically; mid femur with 3-4 av and 3 pv apically; hind femur with 3 av and $2-3$ pv apically. Fore tibia with 1 ad at basal $1 / 4$, apically with comb-like hairs and 3 bristles; mid tibia with 2 ad and 2 pd, apically with 3 bristles; hind tibia with $2 \mathrm{ad}, 4-5 \mathrm{pd}$, and row of $\mathrm{v}$ on whole length, apically with comb-like hairs and 3 bristles. Fore and hind tarsomere 1 with row of short v. Relative length of tibia and 5 tarsomeres of legs LI $4.2: 2.4: 1.0: 0.8: 0.6: 0.3 ;$ LII $5.5: 2.8$ $: 1.5: 1.0: 0.5: 0.4$; LIII $6.4: 2.0: 1.8: 1.0: 0.6$ : 0.5 .

Wing hyaline, veins black; $\mathrm{R}_{4+5}$ and $\mathrm{M}$ parallel apically, CuAx ratio 0.22. Squama yellow with yellow hairs. Halter pale.

Abdomen metallic green with pale gray pollen; hairs and bristles on abdomen black. Male 
genitalia (Fig. 8): Epandrium somewhat rounded, lateral lobe wide with 2 apical hairs, surstylus broad with acute apex; cercus with narrow base and wide apex, with moderately long hairs; aedeagus slender, apically with 1 swollen lateral lobe.

Female. Unknown.

Remarks. The new species is similar to Chrysotus xiaolongmensis Wang \& Yang, 2006, but may be separated from the latter by the completely yellow hind femur and tibia. In xiaolongmensis, the hind femur has the black apex, and the hind tibia is black.

Distribution. China (Xinjiang).

Etymology. The specific epithet refers to the locality of the type Xinjiang.

Acknowledgements. We are grateful to Prof. Chikun Yang, Mrs. Zhu Li, Ms. Kuiyan Zhang, Ms. Hui Dong, Mr. Xingyue Liu, Ms. Yanling Xu, Mr. Changqing Li, and Mr. Xiaoshuan Bai (Beijing), and Prof. Meicai Wei (Changsha) for collecting specimens. The research was supported by the National Natural Science Foundation of China (No. 30225009, 30800106).

\section{References}

Cumming, J. M., Sinclair, B. J. \& Wood, D. M. 1995: Phylogenetic implications of male genitalia in Diptera Eremoneura. - Entomol. Scand. 26: 120-151.

Fallén, C. F. 1823: Monographia Dolichopodum Sveciae. Lundae, 24 pp.

Lichtwardt, B. 1902: Neue ungarische Dolichopodiden (Diptera). - Természetr. Füz. 25: 197-220.

Loew, H. 1857: Neue Beiträge zur Kenntniss der Dipteren.
Fünfter Beitrag. — Programm K. Realschule Meseritz 1857: 1-56.

McAlpine, J. F. 1981: Morphology and terminology adults [Chapter 2]. - In: McAlpine, J. F. Peterson, B. V., Shewell, G. E., Teskey, H. J., Vockeroth, J. R., and Wood, D. M. (eds.), Manual of Nearctic Diptera, Vol. 1. pp. 9-63. Agriculture Canada Monograph, 27, Ottawa.

Meigen, J. W. 1824: Systematische Beschreibung der bekannten europäischen zweiflügeligen Insekten. Vol. 4. Hamm, xii +428 pp.

Negrobov, O. P. 1980: A revision of Palaearctic species of the genus Chrysotus Mg. (Diptera, Dolichopodidae), I. Ch. cilipes $\mathrm{Mg}$. and Ch. laesus Wied. species group. — Entomol. Obozr. 59 (2): 415-420.

Negrobov, O. P. \& Maslova, O. O. 1995: Revision of Palaearctic species of the genus Chrysotus Mg. (Diptera, Dolichopodidae), II. — Entomol. Obozr. 74 (2): 456466.

Negrobov, O. P., Tsurikov, M. N. \& Maslova, O. O. 2000 Revision of the Palaearctic species of the genus Chrysotus Mg. (Diptera, Dolichopodidae), III. - Entomol. Obozr. 79 (1): 227-238.

Negrobov, O. P., Tsurikov, M. N. \& Maslova, O. O. 2003: A revision of the Palaearctic species of the genus Chrysotus Mg. (Diptera, Dolichopodidae), IV. Entomol. Obozr. 82 (1): 223-228.

Robinson, H. \& Vockeroth, J. R. 1981: Dolichopodidae. In: McAlpine, J. F. Peterson, B. V., Shewell, G. E., Teskey, H. J., Vockeroth, J. R., and Wood, D. M. (eds.), Manual of Nearctic Diptera, Vol. 1. pp. 625639. Agriculture Canada Monograph, 27, Ottawa.

Wang, M. Q. \& Yang, D. 2006: Species of Chrysotus Meigen from Beijing (Diptera: Dolichopodidae). - Mitt. Mus. Nat. kd. Berl., Dtsch. Entomol. Z. 52(2): 249255.

Yang, D., Zhu, Y. J., Wang, M. Q. \& Zhang, L. L. 2006: World Catalog of Dolichopodidae (Insecta: Diptera). China Agricultural University Press, Beijing, 704 pp. 